\title{
LOS REGLAMENTOS GEMELOS: EVOLUCIÓN Y MEDIDAS ADOPTADAS ${ }^{1 *}$
}

\author{
Eglè Kavoliūnattė-Ragauskienė \\ Investigadora del Instituto de Derecho del Centro \\ de Ciencias Sociales de Lituania \\ E-mail: egle.kavoliunaite@teise.org
}

\begin{abstract}
RESUMEN: Este trabajo repasa el camino hacia la adopción de los Reglamentos gemelos. En primer lugar, se analizan los argumentos que apoyan la necesidad de una regulación en el ámbito de los bienes matrimoniales y de las parejas registradas. A continuación, se presenta la historia de los intentos de armonizar la regulación de, al menos, algunos aspectos que regulan los bienes matrimoniales. Por último, esboza el procedimiento de elaboración y la adopción del Reglamento de los gemelos.

Palabras clave: Unificación Europea, Derecho de Familia, adopción, Reglamentos gemelos.

ABSTRACT: This paper overviews the road towards the adoption of the Twin Regulations. It firstly discusses the arguments supporting the need for regulation in the area of matrimonial and registered partnership property. It then presents the history of attempts to harmonise regulation for at least some aspects regulating matrimonial property. Finally, the paper outlines the drafting procedure and the adoption of the Twin Regulations.
\end{abstract}

Keywords: European harmonization, European Family Law, adoption, Twin Regulation.

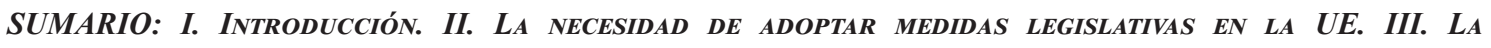

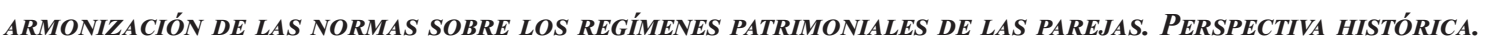

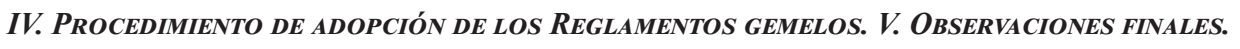

\section{INTRODUCCIÓN}

Durante mucho tiempo la UE no adoptó ninguna medida normativa en el ámbito del derecho de familia, sin embargo, en el siglo XXI se viene desarrollando todo un conjunto de instrumentos de derecho de familia en el ámbito de Derecho internacional privado de la

$\overline{1}$ *Traducido por María José Cazorla González, Profesora Titular de Derecho civil en la Universidad de Almería. 
$\mathrm{UE},{ }^{2}$ siendo algunos de ellos adaptados con relativa facilidad, como son los Reglamentos gemelos que desarrollan el ámbito de los regímenes patrimoniales familiares transfronterizos. Ambas normativas han supuesto un reto, puesto que los efectos patrimoniales del matrimonio y de las uniones registradas quedaban excluidos de normas existentes en la legislación de la UE, como el Reglamento de Bruselas I (artículo 1, apartado 2, letra a)), el Reglamento de Roma I (artículo 1, apartado 2, letra c)), el Reglamento de Roma II (artículo 1, apartado 2, letra b)) y el Reglamento de Roma III (artículo 1, apartado 2, letra e)), era necesaria una regulación respectiva de los regímenes patrimoniales. Esta necesidad fue acentuada en varios programas y documentos marco, así como señalada por el mundo académico.

En respuesta a ello, tras un largo e intenso trabajo, en 2016 se adoptaron el Reglamento en materia de regímenes económicos matrimoniales y el Reglamento sobre las consecuencias patrimoniales de las uniones registradas (conocidos como los Reglamentos gemelos).

Resulta evidente que existen importantes discrepancias entre los Estados miembros de la UE en cuanto al reconocimiento jurídico del estado civil de las parejas, así como diferentes regímenes aplicables a los bienes matrimoniales y de las parejas de hecho, incluso dejando de lado las disposiciones sustanciales del Derecho de familia; por ello, era un reto armonizar la competencia, la ley aplicable y el reconocimiento y la ejecución de las decisiones en este ámbito. En consecuencia, sólo se pudo alcanzar un acuerdo parcial en la UE en forma de cooperación reforzada, lo que significa que no todos los Estados miembros, sino sólo los que lo manifestaron su deseo de participar, están vinculados por estos instrumentos, y sólo una parte de las parejas transfronterizas pueden, por tanto, invocar las normas establecidas en ellos.

Al igual que otros instrumentos de Derecho internacional privado de la UE, los Reglamentos gemelos no armonizan ni modifican ninguna ley nacional sustantiva sobre el matrimonio o la unión registrada. Sólo pretenden establecer normas claras en caso de divorcio o separación y poner fin a procedimientos paralelos y posiblemente conflictivos en los distintos Estados miembros. ${ }^{3}$ Los reglamentos establecen normas por defecto o permiten a las parejas transfronterizas elegir a los tribunales de un Estado miembro concreto para que se pronuncien sobre sus bienes matrimoniales (de pareja) o sobre la ley que se aplica a sus bienes en caso de divorcio, separación o disolución de una pareja registrada. En el caso de las uniones registradas transfronterizas, las normas de la UE aumentan la seguridad jurídica, ya que tienen en cuenta los diferentes enfoques de los Estados miembros en relación con estas formaciones familiares, porque los Reglamentos gemelos aportan generalmente claridad a estas parejas al establecer normas coherentes para determinar qué tribunal del país es competente y qué ley se aplicará, aumentando así la previsibilidad para las parejas al facilitar el proceso de reconocimiento de sentencias, decisiones y títulos en toda la UE.

Este capítulo repasa el camino hacia la adopción de los Reglamentos gemelos. En primer lugar, se analizan los argumentos que apoyan la necesidad de una regulación en el

2 Véase el articulo de la Dra. Agne Limante titulado "El sistema de instrumentos privados de derecho de familia de la UE" en este mismo número de de la Revista Internacional de Doctrina y Jurisprudencia.

3 L. VALENTOVÁ. Property Regimes of Spouses and Partners in New EU Regulations - Jurisdiction, Prorogation and Choice of Law. ICLR, 2016, Vol. 16, No. 2, p. 222. 
ámbito de los bienes matrimoniales y de las parejas registradas. A continuación, se presenta la historia de los intentos de armonizar la regulación de, al menos, algunos aspectos que regulan los bienes matrimoniales. Por último, el capítulo esboza el procedimiento de elaboración y la adopción del Reglamento de los gemelos.

\section{LA NECESIDAD DE ADOPTAR MEDIDAS LEGISLATIVAS EN LA UE}

Durante mucho tiempo, la UE consideró que las cuestiones jurídicas relacionadas con la familia eran responsabilidad de cada Estado miembro. Sin embargo, aprovechando las posibilidades que ofrece el espacio Schengen, las personas empezaron a desplazarse de un Estado a otro de forma más activa, a trabajar en otros países y a iniciar una vida en común con ciudadanos de otro Estado, con los que más tarde deciden casarse o unir su vida cotidiana. ${ }^{4}$ A medida que aumentaba el número de parejas transfronterizas, se hizo evidente en un momento dado la necesidad de una acción comunitaria. Así, durante los últimos veinte años, se han adoptado varios instrumentos de Derecho internacional privado de familia de la UE.

La adopción de los Reglamentos gemelos no fue un caso en el que la legislación se derivara intrínsecamente de la ideología y los documentos primarios de la UE. Fue una solución práctica motivada simplemente por la necesidad de arreglar el resultado que suponía el aumento de la movilidad de las personas y el disfrute de los derechos de libre circulación en el espacio de la UE.

Según el estudio del Consorcio de Evaluación de Políticas Europeas (EPEC) en el que se esbozan diferentes opciones para armonizar los regímenes matrimoniales y de propiedad relacionados con la pareja en la UE, ya en 2007 existían en la UE aproximadamente 122 millones de matrimonios. De ellos, se ha calculado que algo más de 20 millones tenían al menos un tipo de "elementos internacionales" en relación con sus bienes matrimoniales: se suponía que unos 16 millones (el 13\%) eran internacionales; de las que 3,5 millones de parejas casadas vivían en el extranjero (en un país distinto al de su nacionalidad); y alrededor de 1 millón de parejas casadas tenían bienes en el extranjero (en un país distinto al de su residencia habitual).

De forma paralela, se suponía que aproximadamente había 211.000 parejas de hecho registradas en la UE. De ellas, se estimó que algo más de 41.000 tenían algún tipo de "elemento internacional" en relación con sus bienes patrimoniales: se supuso que unas 36.000 uniones registradas eran "internacionales" (es decir, que incluían a parejas de diferentes países), se supuso que unas 2.800 uniones registradas vivían en el extranjero y se supuso que unas 2.500 uniones registradas tenían bienes en el extranjero, dejando así

4 A.P. PÉREZ and M.J.CAZORLA GONZÁLEZ. Matrimonial property regimes in the absence of choice by the spouses under Regulation (EU) 2016/1103. In: M.J. CAZORLA GONZÁLEZ, M. GIOBBI, J.K. ŠKERL, L. RUGGERI, S. WINKLER. Property relations of cross border couples in the European Union. Edizioni Scientifiche Italiane, 2020, p. 29.

5 EUROPEAN POLICY EVALUATION CONSORTIUM (EPEC). Impact Assessment Study on Community Instruments concerning matrimonial property regimes and property of unmarried couples with transnational elements. 2010, pp. 7-13. <https://op.europa.eu/en/publication-detail/-/publication/48820a62-4950-4ebb-a20cd5bc9f35bd84> accessed 22-05-2021. 
constancia que las parejas casadas, las uniones de hecho y las parejas de hecho transfronterizas tenían muchos problemas.

Las parejas transfronterizas se enfrentan a muchos retos cuando se separan. Además del típico estrés psicológico, también tienen que lidiar con las cuestiones legales relacionadas con el hecho de que más de un Estado puede tener jurisdicción para tratar su caso, y que la ley de más de un estado puede ser aplicable. Si bien el Reglamento Bruselas II a, Roma II y otros instrumentos de la UE han dado respuesta a muchas cuestiones, las relativas a los regímenes matrimoniales siguen sin armonizarse.

Uno de los problemas "clásicos" a los que se enfrentan las parejas transfronterizas en Europa, surge del hecho de que el reconocimiento del estatuto jurídico de las parejas en la UE y de la regulación jurídica vinculada a sus vínculos familiares, porque son muy diversos. Tomando como ejemplo una relación entre personas del mismo sexo, en 2015, momento de la iniciativa de los Reglamentos Gemelos, el matrimonio de personas del mismo sexo estaba reconocido en diez Estados miembros de la $\mathrm{UE}^{6}$, y la unión de personas del mismo sexo estaba abierta en diecisiete Estados miembros ${ }^{7}$, donde se incluye a España que aunque carece de ley nacional dispone de regulaciones en algunas Comunidades. Naturalmente, las consecuencias de las uniones registradas difieren significativamente entre los países de la UE, incluso en aquellos que reconocen este tipo de formaciones familiares. Cabe señalar que las iniciativas de unión registrada en los Estados miembros de la UE han surgido como respuesta a una necesidad específica de reconocimiento legal de las parejas del mismo sexo ${ }^{8}$. Así, algunos de los Estados fueron (y siguen siendo) conservadores a la hora de permitir el registro de las parejas de hecho. Aunque la situación legislativa de los países ha cambiado desde 2015, todavía, por ejemplo, seis Estados miembros $^{9}$ no han adoptado aún el modelo de pareja registrada en su legislación y no permiten que las parejas del mismo sexo se casen o se registren su unión. Esto significa que, en lo que respecta a las consecuencias patrimoniales de las parejas del mismo sexo en esos países, no existe ningún régimen jurídico o ley aplicable. Además, en algunos países ${ }^{10}$ el registro de la pareja de hecho está reservado exclusivamente a las parejas del mismo sexo.

Se pueden citar muchos ejemplos sobre la divergencia de normas en el reconocimiento de las distintas formaciones familiares. En Croacia, Eslovenia y Hungría, las normas sobre las consecuencias patrimoniales de las uniones registradas coinciden en líneas generales con las establecidas para los regímenes matrimoniales. En estos países, existe una comunidad de bienes a menos que las partes acuerden lo contrario. Mientras que, en la República Checa, la unión registrada sólo se regula parcialmente similar al matrimonio $\mathrm{y}$, en cuanto a las consecuencias patrimoniales, a diferencia del matrimonio, en Chequia no hay comunidad de bienes para las uniones registradas, si bien las partes pueden adquirir bienes en copropiedad. En Italia, la disciplina de las parejas de hecho registradas refleja la del matrimonio, a pesar de algunas diferencias: por ejemplo, no hay obligación

6 Bélgica, Dinamarca, España, Finlandia, Francia, Luxemburgo, Países Bajos, Portugal, Reino Unido y Suecia.

7 Alemania, Austria, Bélgica, Croacia, Dinamarca, Eslovenia, Estonia, Finlandia, Francia, Hungría, Irlanda, Luxemburgo, Malta, Países Bajos, Reino Unido y República Checa.

8 J.M. SCHERPE. The Past, Present and Future of Registered Partnerships, in: J.M. SCHERPE and A. HAYWARD (eds) The Future of Registered Partnerships. Family Recognition Beyond Marriage?, Cambridge: Intersentia, 2017, p. 570.

9 Bulgaria, Letonia, Lituania, Polonia, Rumanía y Eslovaquia.

10 Croacia, la República Checa, Hungría, Italia y Eslovenia. 
de fidelidad mutua. En cuanto a las consecuencias patrimoniales, las partes están sujetas al régimen de comunidad, como en el caso del matrimonio, salvo que opten por la separación, la pactos patrimoniales y/o la creación del Fondo Patrimonial. ${ }^{11}$

El tercer grupo de Estados miembros permite las uniones registradas de parejas del mismo sexo, pero se aplican normas diferentes a las relativas a las consecuencias patrimoniales del matrimonio. Por ejemplo, en Bélgica y Francia, los efectos de las uniones registradas, aunque son similares a los del matrimonio, no conllevan obligaciones personales entre las partes, que pueden ser del mismo o de distinto sexo. A diferencia del matrimonio, las partes de la unión registrada están sujetas a un sistema de separación de bienes, a menos que acuerden lo contrario. En Chipre, Grecia, Luxemburgo, Malta, los Países Bajos y Estonia, el régimen patrimonial de las parejas registradas es similar al del matrimonio. Y hay cinco Estados miembros (Dinamarca, Finlandia, Alemania, Irlanda y Suecia), en los que las parejas del mismo sexo tienen ahora derecho a contraer matrimonio, si bien anteriormente los miembros de las parejas registradas tenían la opción de convertir la pareja anterior en un matrimonio. ${ }^{12}$

Aunque los instrumentos de la UE no pretenden unificar los diferentes enfoques de los Estados miembros de la UE sobre los matrimonios o las parejas de hecho, sin embargo, las normas de conflicto de leyes a nivel de la UE pueden ayudar a superar al menos algunas incertidumbres legales a las que se enfrentan las parejas transfronterizas debido a esa variedad de leyes nacionales. Por lo tanto, se esperaba que la normativa de la UE en materia de bienes matrimoniales y de pareja fuera especialmente beneficiosa para las parejas al aportar más seguridad jurídica (por ejemplo, estableciendo factores de conexión que no dejen a una pareja transfronteriza en una situación de vacío legal).

Incluso dejando a un lado los diferentes regímenes de unión de los Estados miembros, las diferencias de los Estados miembros en cuanto a las consecuencias patrimoniales del matrimonio y la unión de hecho suponen una gran cantidad de inconvenientes y frustraciones para las parejas transfronterizas, especialmente en relación con la liquidación del régimen económico matrimonial o de la unión de hecho. Para las personas que contraen matrimonio o una pareja de hecho registrada, el problema puede ser la falta de conciencia de que pueden surgir complicaciones legales en el futuro a la hora de gestionar el patrimonio común y la falta de conocimiento de las posibles opciones de organización legal del patrimonio familiar para minimizar la inseguridad jurídica. Para los matrimonios o parejas transfronterizas que se divorcian o separan, surgen muchas preguntas debido a la variedad de leyes nacionales y a la posibilidad de que varias de ellas sean aplicables a su situación. Las diferencias en la legislación aplicable, puede dar lugar a procedimientos paralelos; o a que la gestión de los bienes y su división pueda resultar excesivamente compleja. Incluso cuando se alcanza la solución legal, sin normas comunes de la UE, las decisiones tomadas por un órgano jurisdiccional en un Estado miembro pueden no ser aceptadas en otro. Por úl-

11 R. GARETTO, M. GIOBBI, F.G. VITERBO, L. RUGGERI. Registered partnerships and property consequences. In: M.J. CAZORLA GONZÁLEZ, M. GIOBBI, J.K. ŠKERL, L. RUGGERI, S. WINKLER. Property relations of cross border couples in the European Union. Edizioni Scientifiche Italiane, 2020, p. 89.

12 En Suecia, las uniones registradas ya no se permiten desde 2009, en Dinamarca - desde 2012, en Irlanda - desde 2015, en Finlandia y Alemania - desde 2017. En esas fechas las parejas de hecho tenían la oportunidad de convertir su unión civil en matrimonio, tras la reforma de las normas sobre el matrimonio. En caso de que no lo hicieran, la unión registrada seguía siendo válida en cualquier caso y se seguía aplicando el régimen patrimonial respectivo. GARETTO, M. GIOBBI, F.G. VITERBO, L. RUGGERI, above n. 10, pp. 88-90. 
timo, el cónyuge/pareja superviviente podría verse inmerso en complicados procedimientos judiciales cuando se liquidan los regímenes económicos matrimoniales o el patrimonio de la pareja por el fallecimiento, bien del cónyuge o de un miembro de la pareja. ${ }^{13}$

\section{LAARMONIZACIÓN DE LAS NORMAS SOBRE LOS REGÍMENES PATRIMONIALES DE LAS PAREJAS. PERSPECTIVA HISTÓRICA}

Los primeros documentos jurídicos internacionales que para los matrimonios abordaron cuestiones patrimoniales, fue el Convenio de La Haya de 1905 relativo a los conflictos de leyes relativos a los derechos y deberes de los cónyuges en sus relaciones personales y al ámbito patrimonial; y de otra parte, el Convenio de La Haya de 1978 sobre la ley aplicable a los regímenes económicos matrimoniales. El Convenio de La Haya de 1905 sólo trataba de la ley aplicable a las relaciones personales y patrimoniales de los cónyuges. Las partes de este Convenio fueron Alemania, Bélgica, Francia, Italia, Países Bajos, Polonia (incluida la ciudad libre de Gdansk), Portugal, Rumanía y Suecia. El Convenio entró en vigor en todos los Estados a finales de febrero de 1915, y luego todos los Estados contratantes abandonaron el Tratado en agosto de 1987. Por otra parte, el Convenio de 1978 también se refería exclusivamente a la ley aplicable a los regímenes matrimoniales y se basaba en el principio de universalidad. Sin embargo, aunque el principio de universalidad parecía prometedor para la creación de una cooperación y entendimiento internacional, sólo unos pocos Estados se convirtieron en Estados participantes, ${ }^{14}$ ya que sólo estaba en vigor en Francia, Luxemburgo y Países Bajos, por lo que carecía de efectos internacionales significativos.

La idea inicial de los Reglamentos gemelos surgió del Plan de Acción de Viena de $1998^{15}$ y de su prioridad: la adaptación de las normas sobre regímenes matrimoniales para hacer frente a los problemas derivados de la coexistencia de diferentes leyes y jurisdicciones. Un año más tarde, en 1999, el Consejo Europeo reunido en Tampere respaldó el principio de reconocimiento mutuo de las sentencias y otras decisiones de las autoridades judiciales como piedra angular de la cooperación judicial en materia civil, ${ }^{16}$ e invitó al Consejo y a la Comisión a adoptar un programa de medidas para aplicar dicho principio.

En el año 2000, se elaboró un programa de aplicación del principio de reconocimiento mutuo de las resoluciones en materia civil y mercantil, común a la Comisión y al Consejo. ${ }^{17} \mathrm{El}$ programa identificó claramente la exclusión de importantes sectores del derecho privado del ámbito de aplicación de la normativa europea como uno de los principales obstáculos para la creación de un espacio jurídico europeo, caracterizado por

13 EUROPEAN POLICY EVALUATION CONSORTIUM (EPEC), above n. 4, pp. 9-11.

14 L. VALENTOVÁ, above n. 2, p. 223.

15 CONSEJO EUROPEO, COMISIÓN EUROPEA. Plan de acción del Consejo y de la Comisión sobre la mejor manera de aplicar las disposiciones del Tratato de Amsterdam relativas a la creación de un espacio de libertad, seguridad y justicia - Texto adoptado por el Consejo de Justicia y Asuntos de Interior de 3 de diciembre de 1998 (Diario Oficial n C 019 de 23/01/1999 p. 1)

16 EUROPEAN COUNCIL. Presidency Conclusions. 15-16 October 1999, Tampere. Draft programme of measures for implementation of the principle of mutual recognition of decisions in civil and commercial matters (OJ 2001, C 12, p. 1).

17 EUROPEAN COUNCIL, above n. 15. 
el reconocimiento mutuo de las resoluciones judiciales civiles y mercantiles. Al mismo tiempo, identificó las medidas relativas a la armonización de las normas de conflicto de leyes, como medidas que facilitan el reconocimiento mutuo de las decisiones, y proporcionó principios fundamentales para la elaboración de instrumentos relativos a los testamentos y las sucesiones, a los regímenes matrimoniales y a las consecuencias patrimoniales de la separación de las parejas no casadas.

Pocos años después, el Consejo Europeo celebrado en Bruselas en noviembre de 2004 adoptó un nuevo programa, el Programa de La Haya, en el que se refuerza la libertad, la seguridad y la justicia en la Unión Europea. ${ }^{18}$ El programa invitaba a la Comisión a presentar un Libro Verde sobre el conflicto de leyes en materia de los efectos patrimoniales de las parejas, incluida la cuestión de la competencia y el reconocimiento mutuo, y subrayaba la necesidad de adoptar un instrumento en este último ámbito. En 2006, con la publicación del Libro Verde ${ }^{19}$, la Comisión inició una amplia consulta sobre todos los aspectos de las dificultades a las que se enfrentan las parejas en Europa en relación con la liquidación de sus bienes comunes y los recursos legales disponibles. El Libro Verde también abordó todas las cuestiones de Derecho internacional privado a las que se enfrentan las parejas en uniones distintas del matrimonio, incluidas las parejas con uniones registradas, y las cuestiones específicas para ellas.

A estas acciones anteriores les siguió el Programa de Estocolmo, con una Europa abierta y segura que sirva y proteja a los ciudadanos. ${ }^{20}$ En él, el Consejo Europeo reafirmó la prioridad de desarrollar un espacio de libertad, seguridad y justicia y especificó como prioridad política, la consecución de una Europa de derecho y justicia, también en materia civil. El Programa de Estocolmo consideraba que el reconocimiento mutuo debía extenderse a ámbitos aún no cubiertos pero esenciales para la vida cotidiana, por ejemplo, las sucesiones y los testamentos, los derechos patrimoniales matrimoniales y las consecuencias patrimoniales de la separación de las parejas. También subrayó la necesidad de tener en cuenta los sistemas jurídicos de los Estados miembros, incluido el orden público y las tradiciones nacionales en este ámbito. En el contexto del Programa de Estocolmo, el Consejo Europeo invitó a la Comisión a evaluar si había motivos para la consolidación y la simplificación a fin de mejorar la coherencia de la legislación de la Unión existente en esos ámbitos jurídicos. ${ }^{21}$ Así, el informe sobre la ciudadanía de la UE 2010, eliminó algunos de los obstáculos que afectaban a los derechos de los ciudadanos de la $\mathrm{UE}^{22}$, y que fue presentado por el Parlamento Europeo, quien subrayó la necesidad de una regulación. La petición expresada por el Consejo Europeo y el Parlamento Europeo condujo a la elaboración de diferentes propuestas por parte de la Comisión, entre ellas la Propuesta de Reglamento del Consejo relativo a la competencia, la ley aplicable, el reconocimiento y la ejecución de las resolu-

18 EUROPEAN COUNCIL and EUROPEAN COMMISSION. Action Plan implementing the Hague Programme on strengthening freedom, security and justice in the European Union (OJ 2005, C 198, p. 1).

19 COMMISSION OF THE EUROPEAN COMMUNITIES Green Paper on conflict of laws in matters concerning matrimonial property regimes, including the question of jurisdiction and mutual recognition [SEC(2006) 952], COM/2006/0400 final.

20 EUROPEAN COUNCIL. The Stockholm Programme - An open and secure Europe serving and protecting citizens (OJ 2010, C 115), p. 1.

21 EUROPEAN COMMISSION. EU Citizenship Report 2010: Dismantling the obstacles to EU citizens' rights. [COM $(2010) 603$ final].

22 EUROPEAN PARLIAMENT. Report on the EU Citizenship Report 2010: Dismantling the obstacles to EU citizens' rights $(2011 / 2182(\mathrm{INI}))$. 
ciones en materia de regímenes económicos matrimoniales ${ }^{23}$, y la Propuesta de Reglamento del Consejo relativo a la competencia, la ley aplicable, el reconocimiento y la ejecución de las resoluciones sobre las consecuencias patrimoniales de las uniones registradas ${ }^{24}$ (en lo sucesivo, Propuestas de Reglamentos gemelos). Estos dos documentos dieron lugar a la adopción de los actuales instrumentos jurídicos que se aplican a los regímenes matrimoniales y a las uniones registradas, ambos con implicaciones transfronterizas. ${ }^{25}$

\section{PROCEDIMIENTO DE ADOPCIÓN DE LOS REGLAMENTOS GEMELOS}

El proceso de codificación del Derecho internacional privado europeo suele calificarse de "codificación progresiva". Implica la técnica de adopción de una pluralidad de normas sobre cuestiones bien definidas y limitadas, en lugar de una fuente única aplicable a todo el ámbito. ${ }^{26}$ En este contexto, los Reglamentos gemelos sobre los bienes matrimoniales y los relacionados con las parejas de hecho pretendían unirse a un número creciente de instrumentos de Derecho internacional privado de la UE en materia de Derecho de familia internacional relativos al divorcio y la separación legal (Reglamento Bruselas II a, Reglamento Roma III), a las responsabilidades parentales (Reglamento Bruselas II a), a los alimentos (Reglamento sobre los alimentos) y a las sucesiones y testamentos (Reglamento sobre las sucesiones). ${ }^{27}$

Al redactar los Reglamentos gemelos, las instituciones europeas tuvieron presente la experiencia insatisfactoria del ya mencionado Convenio de La Haya de 1978 sobre la ley aplicable a los regímenes matrimoniales, porque aunque el instrumento parecía prometedor, lo cierto, es que fue ratificado por muy pocos Estados cuando entró en vigor en 1992. El delicado ámbito de la regulación fue probablemente la principal razón del fracaso de este convenio internacional. Las instituciones de la UE también tuvieron que tener en cuenta las restricciones en las competencias legislativas de la UE en cuestiones sustantivas de asuntos familiares, lo que dio lugar a que el objetivo de las nuevas iniciativas legislativas no pudiera ser la armonización de las normas de derecho sustantivo sobre el patrimonio familiar. Además, las tradiciones jurídicas y la regulación del derecho de familia eran muy diferentes en los Estados miembros de la UE, lo que exigía un compromiso en todas las cuestiones.

Por ello, la redacción de los Reglamentos Gemelos no fue una empresa sencilla. Como dicen Garetto, Giobi y otros:

"El camino de la Unión Europea en este ámbito está marcado por obstáculos, bifurcaciones y compromisos provocados por múltiples factores: el fracaso en la elaboración de un

23 EUROPEAN COMMISSION. Proposal for a Council Regulation on jurisdiction, applicable law and the recognition and enforcement of decisions in matters of matrimonial property regimes. [COM(2011) 126 final].

24 EUROPEAN COMMISSION. Proposal for a Council Regulation on jurisdiction, applicable law and the recognition and enforcement of decisions regarding the property consequences of registered partnerships. [COM(2011) 127 final].

25 J.I.SIGNES DE MESA. Introduction. In: M.J. CAZORLA GONZÁLEZ, M. GIOBBI, J.K. ŠKERL, L. RUGGERI, S. WINKLER. Property relations of cross border couples in the European Un-ion. Edizioni Scientifiche Italiane, 2020, p. 6-8.

26 M. CZEPELAK. Would We Like to Have a European Code of Private International Law? 18 European Review of Private Law (2010), pp. 705-728.

27 Véase el capítulo 1 de este volumen sobre la interacción de estos instrumentos. 
Tratado Constitutivo Europeo, la progresiva aparición de fuerzas soberanistas, el Brexit, la extrema fragmentación de las regulaciones domésticas en cuestiones centrales como el reconocimiento de la convivencia, las condiciones exigidas por cada Estado en particular para considerar jurídicamente relevante una determinada convivencia, la previsión de regímenes patrimoniales específicos para las parejas, la identificación de los derechos reales amparados por estas normas, las relaciones en el seno de la pareja y entre la pareja y los terceros acreedores de uno o ambos miembros de la pareja". ${ }^{28}$

Sin embargo, la UE estaba decidida a regular los aspectos transfronterizos de los bienes matrimoniales y los bienes de las parejas de hecho registradas. Más de diez años después del Plan de Acción de Viena de 1998, en marzo de 2011, la Comisión adoptó propuestas de reglamentos gemelos.

A pesar de ello, fracasaron las esperanzas optimistas de que finalmente se aprobaran los instrumentos. Tras las consideraciones del Parlamento y tras recibir su dictamen, en 2015, el Consejo concluyó que no podía alcanzarse la unanimidad para la adopción de las propuestas de reglamentos sobre los regímenes económicos matrimoniales y los efectos patrimoniales de las uniones registradas. Destacó que, en la situación en que se encontraba, los objetivos de cooperación en este ámbito no podían alcanzarse en un plazo razonable por parte de la UE en su conjunto.

Sin embargo, la necesidad de normas comunes se mantuvo. Además del ya mencionado Convenio de La Haya de 1905, relativo a los conflictos de leyes sobre el matrimonio, en lo que les afecta sobre los derechos y deberes de los cónyuges en su relación personal y patrimonial; y del Convenio de La Haya de 1978 sobre la ley aplicable a los regímenes matrimoniales, no hay acuerdos internacionales aplicables a las consecuencias patrimoniales de los matrimonios. En el caso de las parejas de hecho, se aplica el Convenio sobre el Reconocimiento de las Parejas de Hecho, de 5 de septiembre de 2007, de la Comisión Internacional del Estado Civil. Sin embargo, este Convenio sólo abarcaba el reconocimiento de las parejas de hecho, y no había entrado en vigor, por lo que no era probable que ofreciera las soluciones necesarias dada la magnitud de los problemas abordados por los proyectos de Reglamentos gemelos. Por lo tanto, para la Comisión era evidente que, dada la naturaleza y la magnitud de los problemas experimentados por los ciudadanos europeos en los ámbitos de los regímenes matrimoniales y de las parejas de hecho registradas, los objetivos que debían cumplirse con los Reglamentos de Gemelos sólo podían alcanzarse a nivel de la UE. Además, se subrayó repetidamente la necesidad de seguridad jurídica y previsibilidad, exigiendo normas claras y uniformes e imponiendo la forma de reglamentación. De hecho, los objetivos se habrían visto comprometidos si los Estados miembros dispusieran de cierta discrecionalidad a la hora de aplicar estas normas.

Como estaba claro que la regulación del derecho sustancial relativo al estatuto de las parejas y sus regímenes patrimoniales en toda la UE era muy diferente, y algunos Estados miembros que no reconocían los matrimonios y/o las parejas de hecho del mismo sexo estaban preocupados porque, si estaban de acuerdo con la iniciativa, se verían obligados a reconocer dichas uniones. Esto no permitía la unanimidad para seguir adelante con la iniciativa. Por lo tanto, no hubo más remedio que aplicar un modelo de cooperación reforzada para adoptar el Reglamento de Gemelos. Cooperación reforzada que está abierta a todos los

28 R. GARETTO, M. GIOBBI, F.G. VITERBO, L. RUGGERI, above n. 10, p. 43. 
Estados miembros, siempre que cumplan las condiciones de participación establecidas en la decisión de autorización. También está abierta a ellos en cualquier otro momento, siempre que se respeten los actos ya adoptados en ese marco, además de las condiciones establecidas.

En junio de 2016, tras muchos retrasos y obstáculos, los Reglamentos gemelos se adoptaron finalmente mediante el procedimiento de cooperación reforzada. Actualmente, los Reglamentos gemelos son obligatorios en su totalidad y directamente aplicables solo en los Estados miembros que participan en la cooperación reforzada definida en virtud de la Decisión (UE) 2016/95429, es decir, Bélgica, Bulgaria, Chipre, la República Checa, Alemania, Grecia, España, Francia, Croacia, Italia, Luxemburgo, Malta, los Países Bajos, Austria, Portugal, Eslovenia, Finlandia y Suecia.

Sin embargo, incluso con la cooperación reforzada, podemos constatar que la adopción de los reglamentos gemelos requirió un compromiso. El Reglamento sobre los regímenes económicos matrimoniales no define el concepto de "matrimonio", que se deja al arbitrio de las legislaciones nacionales de los Estados miembros. En cambio, el Reglamento sobre las consecuencias patrimoniales de las uniones registradas establece un concepto de "unión registrada", que se define únicamente a efectos del reglamento. El contenido real del concepto queda definido en las legislaciones nacionales de los Estados miembros. Nada en el Reglamento sobre las consecuencias patrimoniales de las uniones registradas obliga a un Estado miembro cuya legislación no contemple la institución de la unión registrada a preverla en su legislación nacional. Este planteamiento revela el sólido compromiso de la UE de respetar los sistemas nacionales de derecho de familia. Sin embargo, dado que el modo en que se contemplan las formas de unión distintas del matrimonio en la legislación de los Estados miembros difiere de un Estado a otro, el Reglamento sobre las consecuencias patrimoniales de las uniones registradas establece una distinción entre las parejas cuya unión está sancionada institucionalmente y aquellas otras parejas de hecho que no están registradas. Por ello, el Reglamento sobre las consecuencias patrimoniales de las uniones registradas sólo considera las uniones registradas que tienen carácter oficial a los efectos de las normas que establece. ${ }^{30}$

Dado que la UE carece de competencias para interferir en el derecho sustantivo, en el ámbito del derecho de familia, los esfuerzos de la UE se han dirigido a la creación de un marco uniforme de normas de conflicto de leyes destinado a resolver aquellas cuestiones familiares que tienen implicaciones transfronterizas. ${ }^{31}$ Por lo tanto, los Reglamentos gemelos prevén una cooperación reforzada en tres cuestiones básicas: (i) la determinación del tribunal competente, (ii) la determinación de la ley aplicable, y (iii) el reconocimiento y la ejecución de las sentencias sobre los efectos patrimoniales de las parejas transfronterizas. Cabe señalar que el Reglamento de los gemelos subraya repetidamente la necesidad

29 EUROPEAN COUNCIL. Decisión (UE) 2016/954, de 9 de junio de 2016, por la que se autoriza una cooperación reforzada en el ámbito de la competencia judicial, la ley aplicable y el reconocimiento y la ejecución de resoluciones sobre los regímenes patrimoniales de las parejas internacionales, que abarca tanto las cuestiones relativas a los regímenes matrimoniales como los efectos patrimoniales de las uniones registradas. OJ 2016, L 159, p. 16.

30 J.I.S DE MESA, above n. 24, pp. 9-10.

31 A.M.P. VALLEJO. Matrimonial property regimes with cross-border implications: Regulation (EU) 2016/1103. In: M.J.C. GONZÁLEZ, M. GIOBBI, J.K. ŠKERL, L. RUGGERI, S. WINKLER. Property relations of cross border couples in the European Union. Edizioni Scientifiche Italiane, 2020, p. 15.; M.J.C. GONZALEZ. Ley aplicable al régimen económico matrimonial después de la disolución del matrimonio tras la entrada en vigor del Reglamento UE 2016/1104. 21 International Journal of Doctrine and Jurisprudence (2019), pp. 87-104. 
de previsibilidad y seguridad jurídica, ya que es importante que los cónyuges y las parejas conozcan cuál es el tribunal que será competente para resolver las cuestiones patrimoniales y qué ley les será aplicable. A este respecto, el Reglamento también da un amplio margen a la autonomía de la voluntad a la hora de hacer esta elección. ${ }^{32}$

Los Reglamentos gemelos no modifican las normas de cada Estado miembro, sino que contribuyen a determinar la competencia y la ley aplicable al régimen económico matrimonial de los cónyuges que hayan contraído matrimonio a partir del 29 de enero de 2019 y hayan decidido elegir la ley aplicable en virtud del artículo 22. En otras palabras, la aplicación del Reglamento supone un cambio en las normas nacionales sobre la resolución de litigios "internacionales" o "transfronterizos", en lo que respecta a su ámbito de aplicación material y temporal. ${ }^{33} \mathrm{El}$ buen funcionamiento de un espacio común de justicia, que respete los diferentes sistemas y tradiciones jurídicas de los Estados miembros, es uno de los objetivos de la Unión Europea. En este sentido, el Reglamento, continuando la tradición de los instrumentos jurídicos precedentes, constituye un nuevo avance hacia la unificación del Derecho internacional privado de familia en Europa.

La adopción de los reglamentos gemelos fue muy bien recibida en el ámbito político de la UE. Sin embargo, se planteó una gran preocupación por el hecho de que un número importante de parejas sigue quedando fuera del ámbito de aplicación de los Reglamentos. Los 18 Estados miembros que se adhirieron a la cooperación reforzada constituyen el 70\% de la población de la UE y representan la mayoría de las parejas internacionales que viven en la UE. ${ }^{34}$ Los Estados miembros no participantes siguen aplicando sus respectivas legislaciones nacionales (incluidas sus normas de Derecho internacional privado) a las situaciones transfronterizas relacionadas con los regímenes matrimoniales y las consecuencias patrimoniales de las parejas de hecho. A este respecto, el vicepresidente primero de la Comisión Europea, Frans Timmermans, señaló:

"Se trata de dar seguridad a miles de parejas europeas, casadas o registradas, sobre lo que les ocurre a ellos y a sus familias cuando el sueño de una vida en común no funciona. Ojalá hubiéramos podido llevar esto adelante con todos los Estados miembros, como había propuesto la Comisión, pero las propuestas de hoy significan que podemos ayudar al menos a algunas de las personas para que dispongan de instrumentos jurídicos que les ayuden a gestionar y resolver problemas en momentos difíciles."

Del mismo modo, Vera Jourová, Comisaria de Justicia de la UE, observó que:

"En caso de divorcio o fallecimiento de la pareja, la vida de 16 millones de parejas internacionales puede complicarse aún más debido a los engorrosos trámites administrativos y a la falta de claridad de las situaciones jurídicas: ¿qué pasará con mi casa si me divorcio y mi cónyuge es de otra nacionalidad? ¿A qué tribunal me dirijo? Las nuevas normas propuestas aportarán claridad jurídica y facilitarán el complicado proceso de reparto de los bienes comunes, independientemente de dónde se encuentren. Esto facilitará la vida de las parejas afectadas y les ayudará a ahorrar unos 400 millones de euros al año de costes adi-

32 A.M.P. VALLEJO above n. 34, p. 15.

33 P.C.G DE PARADA. Nuevos reglamentos europeos sobre regímenes matrimoniales y sobre efectos patrimoniales de las uniones registradas. 84 El Notario del Siglo XXI, (2019).

34 EUROPEAN COMMISSION. Property regimes for international couples in Europe: new rules apply in 18 Member States as of today. $<$ https://ec.europa.eu/commission/presscorner/detail/en/IP 19_681> accessed 22-05-2021. 
cionales. Hoy allanamos el camino para aquellos Estados miembros que estén dispuestos a seguir adelante con esta importante iniciativa". ${ }^{35}$

De hecho, en este contexto es un gran reto, unificar las disposiciones europeas que buscan un enfoque normativo con más sinergias en los que a regímenes económicos matrimoniales se refiere, porque la unificación y simplificación de las normas en el ámbito de las consecuencias patrimoniales de las uniones registradas, como se ha comentado anteriormente, es un objetivo aún más difícil.

\section{OBSERVACIONES FINALES}

La libre circulación de personas y la posibilidad de trabajar en cualquier país de la UE son a la vez una oportunidad y un reto para los ciudadanos y las parejas de la UE. Aunque parezca paradójico, el mayor grado de libertades personales conlleva la necesidad de una mayor regulación, ya que los nuevos estilos de vida no se ajustan a los marcos legales existentes. La legislación nacional que regula los asuntos familiares es insuficiente, especialmente cuando se trata de situaciones de conflicto o de finalización de una relación. Por lo tanto, son necesarias ciertas normas de conflicto que regulen la aplicación de las normas sustanciales nacionales, y la adopción de instrumentos en la UE.

Aunque, en teoría, no parece tan difícil trazar una línea divisoria entre las normas sustantivas de derecho de familia y las referidas al procedimiento de naturaleza procesal, la cuestión patrimonial plantea muchos retos. En primer lugar, debemos tener en cuenta los 27 Estados de la UE donde se regula de forma diferente la división de los bienes conyugales, lo que dificulta no solo la creación de las normas, sino también el proceso de adopción de las mismas, dificultad que aumenta cuando los bienes que deben dividirse pertenecen a las parejas registradas. ${ }^{36}$ En segundo lugar, es difícil separar las normas puramente procesales de las normas materiales (sustantivas) de Derecho de familia, ya que, a este respecto, son muy interdependientes. Las normas procesales dependen también del estatuto jurídico de la pareja, por ejemplo, en caso de que un país no reconozca la unión registrada, la aplicabilidad del Reglamento sobre las consecuencias patrimoniales de las uniones registradas resulta imposible.

El hecho de que el proceso de adopción de los reglamentos gemelos haya durado más de diez años, y de que estos reglamentos sólo sean aplicables a una parte de las parejas de la UE, podría parecer poco prometedor. Sin embargo, este paso debe percibirse como un logro y un avance, sobre todo teniendo en cuenta los anteriores esfuerzos infructuosos por armonizar las normas procesales internacionales en el pasado. Cada vez son más los Estados miembros de la UE que adoptan regímenes liberales sobre el estatuto jurídico de las parejas y, teniendo en cuenta que cualquier Estado miembro puede incorporarse al marco del Reglamento sobre los gemelos, esto implica que cabe esperar una mayor evolución y una ampliación del ámbito de aplicación.

35 EUROPEAN COMMISSION. Commission goes ahead with 17 Member States to clarify the rules applicable to property regimes for Europe's international couples. $<$ https://ec.europa.eu/commission/presscorner/detail/en/ IP $16 \quad 449>$ accessed 22-05-2021.

36 L. VALENTOVÁ, above n. 2, p. 222. 